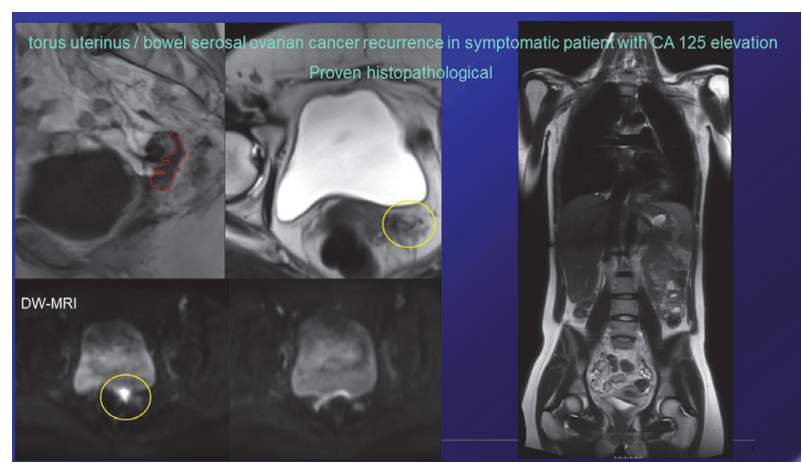

Abstract 554 Figure 1

tumor detection (Tu-Det). Furthermore, three patients were imaged having uterine, cervical and gestational breast cancer. Result(s)* 5/6 (REC), 5/10 (F-up), 4/6 (Tu-Det) showed pathologic diffusion restricted MR-graphic findings. Even MRgraphic only faint $\mathrm{T} 2 \mathrm{w}$ but diffusion restricted bright lesions were verified to be carcinomatous intraoperative/histopathological. Apparent diffusion coefficient (ADC) values were calculated. Primary and metastatic tumor sites were reported. When operated, the MR-graphic findings were correlated with intra-operative and histopathological findings.

Conclusion* DW-MRI without contrast media is a feasible and sensitive tool in OC primary diagnostics, staging and in detecting recurrence. Change is difficult, but sometimes necessary, as also former studies of this topic show. Larger multicenter studies are required in order to investigate the impact of DW-MRI for therapy management and overall survival.

\section{NON-BRCA1/2 PATHOGENIC/LIKELY PATHOGENIC VARIANTS DETECTED IN $2.6 \%$ OF PATIENTS WITH OVARIAN, FALLOPIAN TUBE OR PRIMARY PERITONEAL CANCER}

\footnotetext{
${ }^{1}$ VA Mesaric*, ${ }^{1} \mathrm{~K}$ Drusany Staric, ${ }^{2} \mathrm{~S}$ Hotujec, ${ }^{2} \mathrm{~K}$ Strojnik, ${ }^{2} \mathrm{~A}$ Blatnik, ${ }^{2} \mathrm{M}$ Banjac, ${ }^{3} \mathrm{E}$ Skof, ${ }^{4} \mathrm{~V}$ Stegel, ${ }^{4} \mathrm{~S}$ Novaković, ${ }^{2} \mathrm{M}$ Krajc. ${ }^{1}$ University Medical Center Ljubljana, Department of Gynaecology, Ljubljana, Slovenia; ${ }^{2}$ Institute of Oncology Ljubljana, Cancer Genetics Clinic, Ljubljana, Slovenia; 3 Institute of Oncology Ljubljana, Division of Medical Oncology, Ljubljana, Slovenia; ${ }^{4}$ Institute of Oncology Ljubljana, Department of Molecular Diagnostics, Ljubljana, Slovenia
}

\subsection{6/ijgc-2021-ESGO.428}

Introduction/Background* In Slovenia, ovarian cancer is diagnosed in approximately 160 women per year. The majority of patients are diagnosed with advanced disease and the survival rate is poor. $20-30 \%$ of cases can be attributed to germline pathogenic/likely pathogenic variants $(\mathrm{P} / \mathrm{LPV})$ in genes, associated with hereditary breast and ovarian cancer syndrome (HBOC). P/LPV in BRCA1/2 genes are the most prevalent. With the development of new methods for genetic testing, such as next generation sequencing (NGS), associations between non-BRCA genes and HBOCare being discovered.

Methodology We analyzed genetic results of patients with the diagnosis of ovarian, fallopian tube or primary peritoneal cancer, who were concecutively referred for genetic assessment at our institution in the period between September 2014 and April 2021. Since September 2014 all such patients are routinely tested with NGS with a panel (TruSight Cancer panel

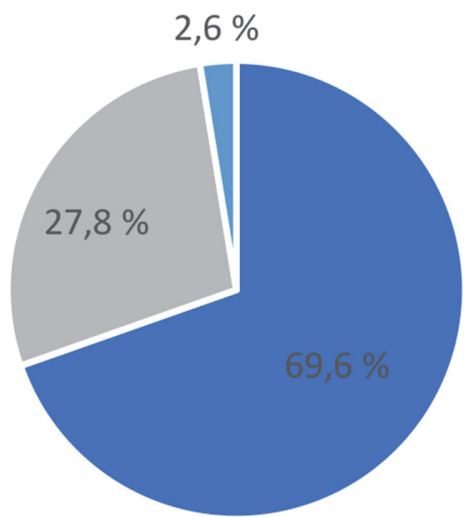

\section{- Negative - BRCA1/2 " Other than BRCA1/2}

Abstract 558 Figure 1 Results of genetic testing of patients with ovarian, fallopian tube or primary peritoneal cancer

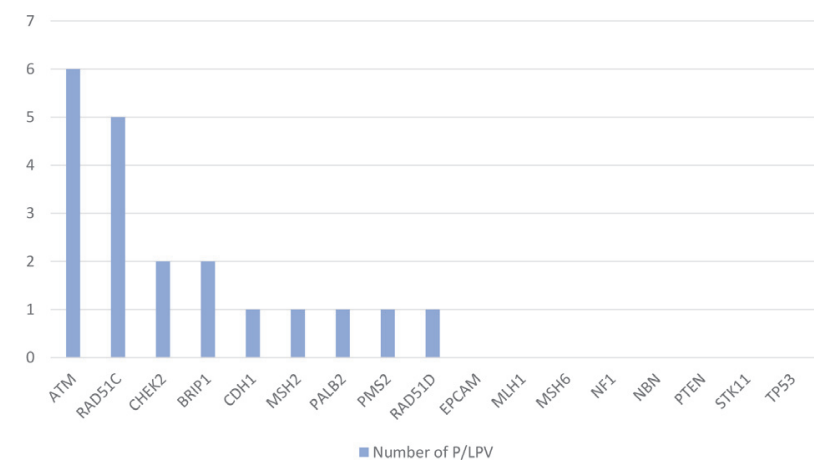

Abstract 558 Figure 2 Non-BRCA1/2 mutations in patients with ovarian, fallopian tube or primary peritoneal cancer, tested with our HBOC panel

or TruSight Hereditary Cancer panel) of 19 HBOC genes. In some cases, an analysis of tumor tissue was also performed.

Result(s)* During the observation period, 744 patients were tested for germline $\mathrm{P} / \mathrm{LPV}$ in HBOC gene panel. P/LPV was found in 226/744 (30.4\%) of tested patients. 207/744 (27.8\%) of patients had P/LPV in BRCA1/2. Non-BRCA P/LPVs were found in 19 patients (2.6\%), one patient had a P/LPV in both $A T M$ and $R A D 51 C$ genes (figure 1). The most common $\mathrm{P} /$ LPVs in non-BRCA genes were detected in ATM gene, and were diagnosed in 6 patients (figure 2). The results of tumor tissue testing were available for 7/19 nonBRCA-positive patients and in 1 biallelic PALB2 inactivation was found.

Conclusion* Among 744 consecutively tested patients with ovarian, fallopian tube or primary peritoneal cancer, we detected a high $\mathrm{P} / \mathrm{LPV}$ rate $(30.4 \%) .27 .8 \%$ of all tested patients were diagnosed with $B R C A 1 / 2$ P/LPVs. P/LPVs in other than $B R C A 1 / 2$ genes were detected less frequently (2.6\%). It is, however, important to be aware that P/LPVs in genes other than BRCA1/2can be detected in patients with ovarian cancer and that these women may also benefit from targeted treatment, preventive measures and further cascade testing in the family. Tumor testing may also reveal new treatment targets and help explain carcinogenesis in these patients. 